\title{
Source-Parameter Estimation in a Coal Environment
}

\author{
A.R. King CSIRO Exploration and Mining, Australia
}

\begin{abstract}
Microseismic surveys conducted in coal environments are subject to a unique set of problems: (1) Coal seams have a large velocity and density contrast with the host rocks, and so strong, reflections and mode-conversions are present in the seismic traces, often overlapping with the direct arrival. This means that separation of source and propagation effects is critical if source parameters are to be accurately determined. (2) The finely-layered nature of the sedimentary medium results in strong S-wave splitting, with velocity differences of the order of $25 \%$ between SV and SH polarizations. (3) The low soft-rock velocities mean that mine-scale seismic observations are often in a region where the near-field effects are significant. This changes the shape of the seismic velocity traces, but also provides extra information useful for determining source parameters.
\end{abstract}

This paper shows examples of these effects from longwall coal mines in Australia, and discusses methods of sourceparameter identification in their presence.

\section{INTRODUCTION}

Microseismic monitoring in a coal longwall mining environment is concerned with caving that is progressive, with fracturing occurring some distance ahead of the longwall face as it advances. Departures from a "normal" state of seismic activity about the face are of interest, because they indicate potential problems, such as stronger roof "hanging up", and leading to excessive weighting on chocks, or wind blast caused by mass falls of roof.

Seismic activity is routinely detected in areas where the predicted stresses are much lower than the rock strength. Events are generally detected far ahead of the face, in regions where the abutment stress is predicted to have had only a small effect on in situ stress conditions, and the seismic events then increase in number as the face approaches. This same mechanism has been observed in laboratory-scale acoustic emission experiments.

In order to more closely link the seismic observations with geotechnical predictions, it is desirable to be able to characterize the seismic source, and extract parameters such as fracture mode, size, orientation and stress drop. This information would then enable different kinds of potentially interesting fractures to be distinguished: those on bedding planes from those through intact rock, or shear fractures from tensile fractures, for example.

It is common to assume a homogeneous velocity model when processing microseismic data. However, in a coal environment this can lead to serious problems in interpreting the data, even though the coal seams - the only appreciable departure from uniformity - are generally relatively thin. Moment tensor inversions of events in a coal environment often produce inconsistent results. Inversions using only $\mathrm{P}$-wave arrivals fail to predict the observed S-waves, often predicting much larger amplitudes than observed. And inversions making use of both $\mathrm{P}$ and $\mathrm{S}$ waves result in very much larger fitting errors than would be expected from the data quality. The first question to be asked is: what is the reason for these poor moment tensor results?

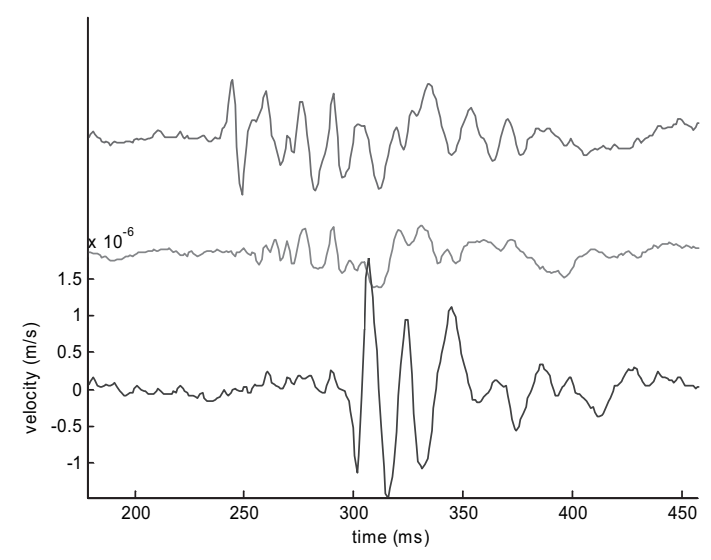

FIG. 1 A typical seismogram exhibiting the lack of a clear SV arrival. From top to bottom, the traces show P, SV, SH components

Another question concerns the common occurrence of waveforms with very small S-wave arrivals. In particular, many seismograms are seen with a significant $\mathrm{SH}$ arrival, but small, or nonexistent SV arrivals. This is seen far more often than could be explained by the presence of null points in the $\mathrm{S}$-wave radiation pattern of a shear event. An example of such a seismogram is shown in Fig. 1, where the seismogram has been rotated into a P-SV-SH coordinate system. Are these waveforms due to unusual source mechanisms, or are they propagation effects?

\section{DATA}

The data presented here were all acquired with the CSIRO's microseismic system, which was developed in-house. Details of the system, and examples of data recorded are given in (Hatherly et al., 1997). Geophones are used as sensors, due to the low dominant frequencies of the data. The waveforms are sampled at a frequency between 1 and $2 \mathrm{kHz}$, typically for a duration of one second.

The acquisition geometry normally consists of 15-20 triaxial geophones, arranged in 3 or 4 vertical boreholes, although occasionally surface geophones are also employed. 
Longwall mining panels are typically $150 \mathrm{~m}$ to $250 \mathrm{~m}$ wide, and the horizontal distance between boreholes containing geophone strings is normally of a similar size. The vertical span of the geophone arrays is typically around $100 \mathrm{~m}$, so the focal sphere of events within the array is reasonably well sampled. Low-angled rays, however, do tend to dominate, except for events which are close to one of the geophone strings.

\section{EFFECTS OF COAL SEAM REFLECTIONS}

The coal environment is unique in the strength of the contrast between the coal seams and the surrounding sandstones and shales. Seismic P-wave velocities in the sandstones are generally around $3500-3900 \mathrm{~m} / \mathrm{s}$, whereas in the coal the velocities are down around $2500 \mathrm{~m} / \mathrm{s}$. The contrast in density is also large, from $2.6 \mathrm{~g} / \mathrm{cm}^{3}$ in the sandstones to $1.3 \mathrm{~g} / \mathrm{cm}^{3}$ in the coal. This means that the coal seams are extremely strong seismic reflectors. To illustrate the strength of the coal-seam reflections, a plot of reflection and transmission amplitudes versus angle of incidence for $\mathrm{P}$-waves passing from sandstone into coal is shown in Fig. 2.

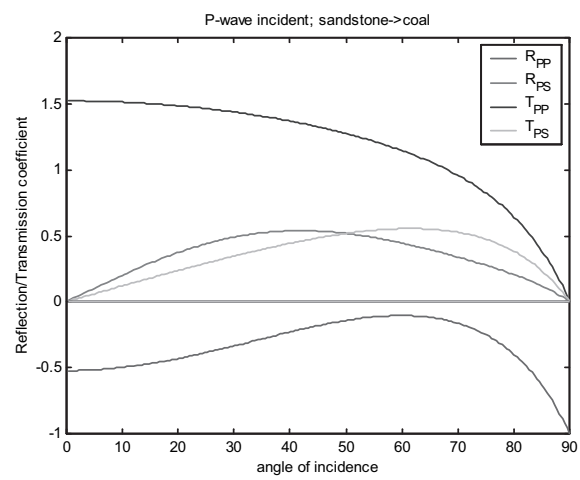

FIG. 2 Reflection and transmission coefficients for a P-wave arriving at a sandstone-coal interface

The coal seams are also close enough to the majority of the mining-induced events that the reflections overlap the direct arrivals. This means that an apparent arrival is in fact made up of a series of overlapping reflected arrivals. And, rather than representing the strength of the radiation pattern in one particular direction, it is in fact sampling the radiation pattern at many, possibly very different, departure angles.

Some synthetic examples will illustrate the problem.

Fig. 3 shows a synthetic reflection off a thick coal seam, where internal reverberations are neglected. The top of the coal seam is shown, as well as source and receiver positions, and the ray paths of the various reflected and converted modes. The arrival times of the rays, relative to the P-wave, are also shown, from which it can be seen that there are three reflected arrivals which would overlap a P-wave of typical pulse width. The relative amplitudes of the outgoing rays can be seen by examining the cross-section through the radiation pattern: The direct $\mathrm{P}$ wave is close to a nodal plane, and has small amplitude, while the SP refected arrival leaves at an angle which has close to the maximum S-wave amplitude. This is a worst-case scenario, where the geophone lies close to the nodal plane of the P-wave radiation pattern, and where, in the presence of noise, the reflected SP converted wave is likely to be mistakenly identified as the direct $\mathrm{P}$ arrival.

Numerous thin coal seams are often present in the vicinity of the seam being mined. Although these thin seams have minimal effect on near-vertical propagation, seismograms recorded at a near-horizontal location relative to the source can be quite dramatically affected. The series of multiple internal reflections in the seam can interfere significantly
B3

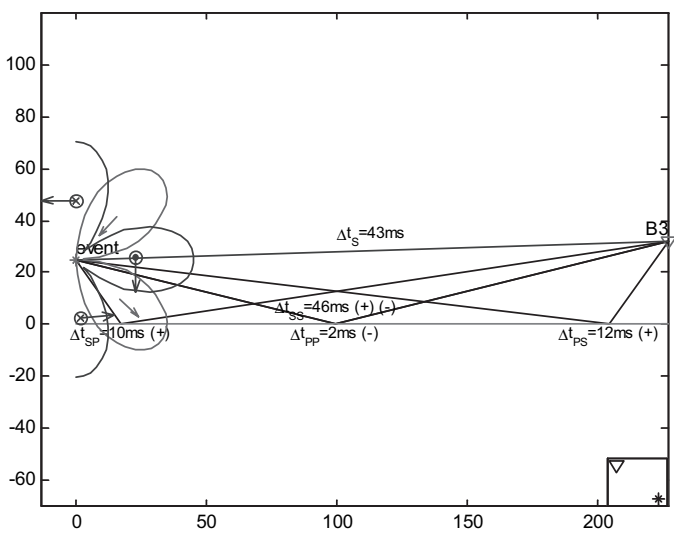

(a)

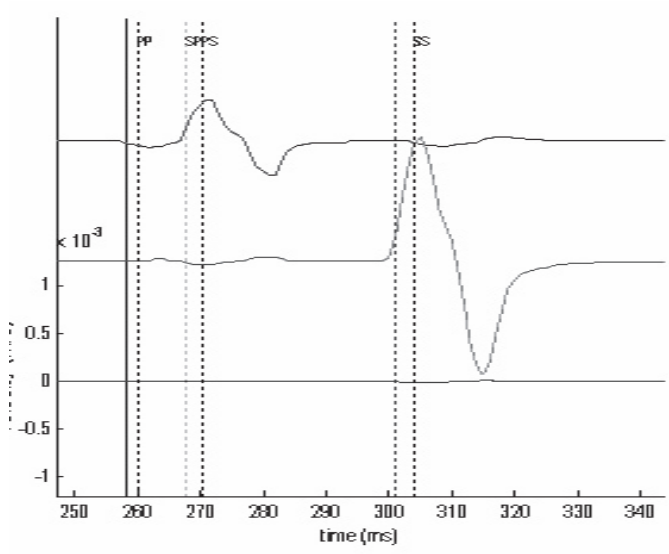

(b)

FIG. 3 Effect of reflection from a thick seam. The geometry shown in (a) includes locations of the source event, geophone, coal-seam top, and the converted rays. The arrival times of the various modes, relative to the P-arrival, is also indicated. The resulting synthetic waveform is shown in (b)

with the direct SV arrival, as illustrated by the example in Fig. 4. The dashed lines show the recorded seismogram in the absence of a coal seam, while the solid lines show the seismogram in the presence of a nearby, $1 \mathrm{~m}$-thick seam. The effects of anisotropy have been included, thereby shifting the $\mathrm{SV}$ and SH arrivals. It can be seen that the SV arrival has been cancelled by the internal seam reverberations. Modelling has shown this to be a relatively common effect in these low-angle circumstances, thereby explaining the observations of missing $\mathrm{S}$-wave arrivals on otherwise normal seismic traces. The $\mathrm{P}$ wave has also been dramatically affected by the presence of overlapping arrivals, having an apparent amplitude twice as great as its true amplitude.

This example shows rays passing through a thin coal seam, but the same effect is observed when source and receiver are on the same side of the seam.

By contrast, Fig. 5 shows the effects of low-angle transmission through a thicker seam, $5 \mathrm{~m}$ thick in this example. This is the same geometry, and the same source mechanism as in the previous example - only the seam thickness has been changed. Here the internal seam reflections can be clearly seen in the traces. 


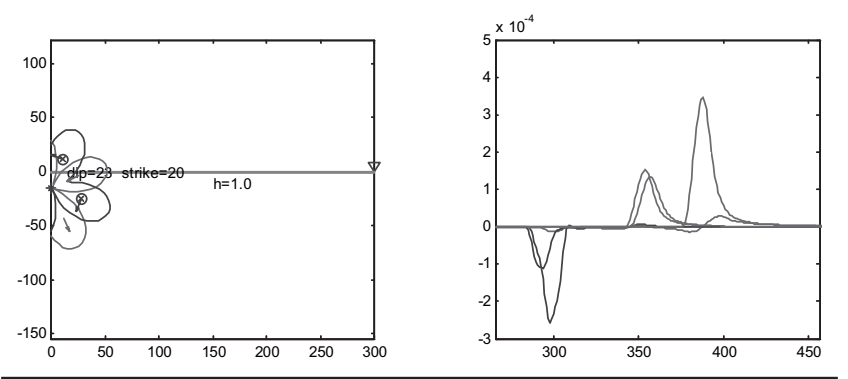

FIG. 4 The effect of low-angle transmission through a thin coal seam

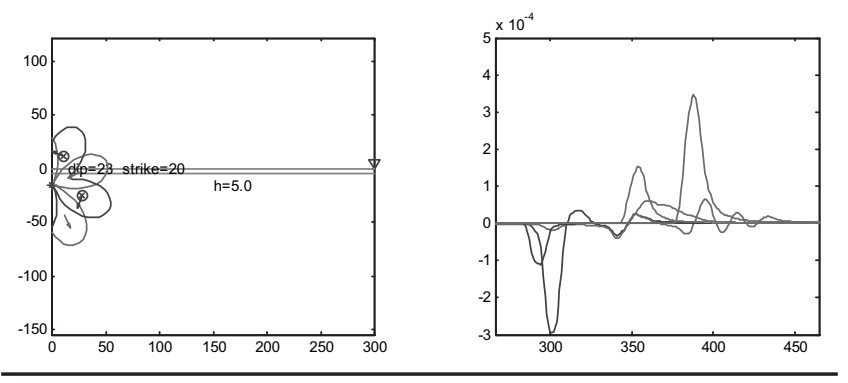

FIG. 5 The effect of low-angle transmission through a thick seam

\section{THE NEAR FIELD}

The combination of small distances to the source, of hundreds of meters, and the relatively low velocities of a soft-rock environment, means that near-field effects are clearly visible on the displacement seismograms. Near-field effects have seldom been regarded as important in a mining-seismicity context (though see (McGarr, 1992a; McGarr, 1992b) for examples) but in this context turn out to be quite significant. Fig. 6 shows an example seismogram, overlaid by a synthetic fit including the near-field terms modelled using the expressions given in (Aki and Richards, 1980). No reflections from coal seams have been modelled, and anisotropy has not been included. The effect is most easily seen on the $\mathrm{SH}$ trace, where the near field response can be seen in the absence of any interfering reflected arrivals. The various components of the field are plotted in light grey dotted lines, from which it can be seen that the near-field terms are comparable in magnitude to the far-field terms at this distance, and so need to be included in any source parameter inversion.

On the other hand, the coal environment is close to being perfectly horizontally layered, and so the $\mathrm{SH}$ trace of a seismogram contains no reflected arrivals prior to the direct $S$ arrival. This means that the near-field ramp observed here is the "cleanest" information available on the source pulse itself, and hence supplies potentially useful additional information relevant to source-parameter determination.

\section{ANISOTROPY AND S-WAVE SPLITTING}

Examination of geophysical borehole log data from a coal environment shows the presence of very fine layering in the sedimentary sequence, with corresponding changes in seismic velocity as the rock types vary between sandstones and shales. The scale of this layering, going down to tens of centimetres or less, is significantly smaller than the seismic wavelengths of interest, which are in the range of metres to tens of metres. The result is the presence of a strong, rotationally symmetric anisotropy in the seismic propagation characteristics of the rocks.

An example seismogram, rotated into P-SV-SH coordinates, is shown in Fig. 7. Here it can be seen that the arrival time

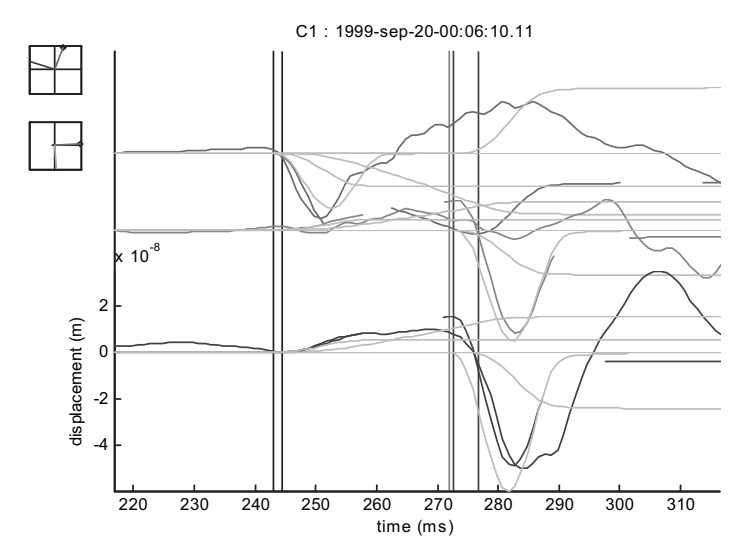

FIG. 6 Near-field effects: The observed displacement seismogram (solid) is overlaid with a synthetic seismogram (dashed) including the near-field terms

difference between the SH and SV modes is almost as great as that between $\mathrm{P}$ and $\mathrm{SH}$. This example, with the source at a distance of $130 \mathrm{~m}$, and a $20 \mathrm{~m}$ vertical separation, has a difference of about $25 \%$ between the $\mathrm{SV}$ and $\mathrm{SH}$ propagation velocities. The $\mathrm{SH}$ velocity usually seems to lie close to the Poisson-solid value of $\alpha / \sqrt{3}$, while the SV velocity is typically $25 \%$ slower, at least for the low-angle rays common with this survey geometry.

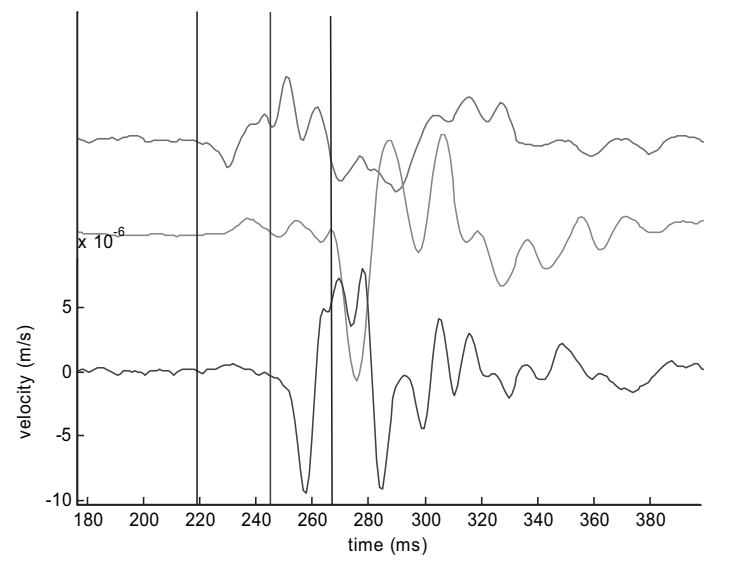

FIG. 7 S-wave splitting: A displacement seismogram, rotated into P-SV-SH coordinates

\section{DISCUSSION}

The main problem that triggered this work was that naïve moment-tensor inversions failed. Inverted moment tensors that resulted in reasonable fits to the P-wave arrival data turned out, on closer inspection, to predict totally incorrect amplitudes for the S-wave arrivals. Attempts to correct the situation by inverting for all arrivals simultaneously were unable, in the main, to arrive at consistent results.

Correctly modelling and removing propagation effects from the seismic waveforms is essential if the source time function is to be recovered. This in turn is required for any moment tensor inversion or other source parameter determination. The interesting questions to ask are: how much of the complexity of the waveform is due to propagation effects, and how much of it is inherent in the source? Which propagation effects are important, and which can be neglected?

Reflections of seismic waves off coal seams are clearly important. A quick plot of the reflection coefficients for a sandstone-coal interface with average seismic parameters 
shows that the reflection amplitudes are likely to be large. Most of the mining-induced microseismic events occur in the immediate roof of the coal seam being mined, up to a few tens of metres. So the difference in travel time of the direct rays and the rays reflected from the top of this seam is of the order of a few milliseconds. Since the seismic pulse widths visible on the seismograms are typically around $10-30 \mathrm{~ms}$, this means that most of the arrival pulses visible on the seismic traces will in fact be composed of a series of overlapping reflections, along with the direct ray. No sensible attempt at source-parameter determination can be made without accounting for these reflections.

Near-field effects, while not generally as strong in amplitude as the coal reflections, are large enough to affect the results, and so should be modelled for this kind of data. The radiation patterns of the near-field terms are different from those of the $\mathrm{P}$ and $\mathrm{S}$ waves, and so provide independent information on the source mechanism. Example radiation patterns of the different components for a shear event are shown in Fig. 8, illustrating these differences.
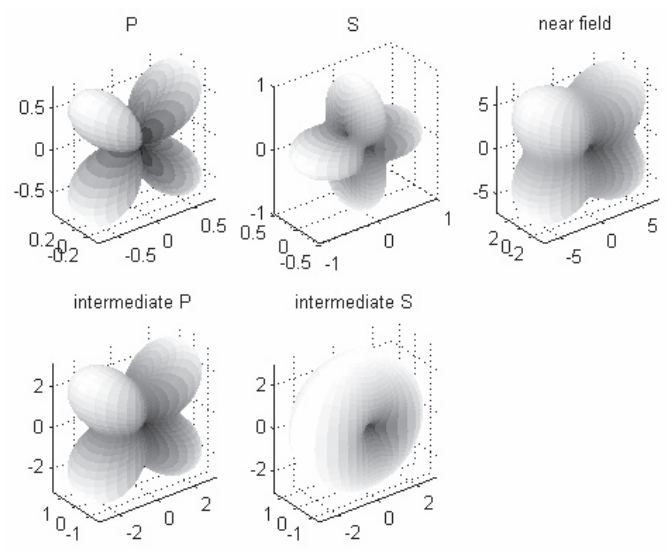

FIG. 8 Radiation patterns for far-, intermediate-, and near-field terms for a vertically dipping shear fracture

Inversion for moment tensor components and other source parameters is a much more complex procedure when reflections are taken into account. This is because the degree of fit between an observed and a synthetic seismogram depends crucially on whether the various phase arrival times have been correctly computed. Errors in the location of the seismic event cause errors in the relative time of arrival of the reflections. This means that the algorithm needs to be able to somewhat vary the event locations in order to fit the details of the waveform data. In addition, seismic velocities vary over the volume where events are being sampled, and the coal-seam reflectors are not perfectly layered. These factors have similar effects on the relative pulse arrival times.

Since our ultimate aim is to determine the source parameters, rather than to characterize the rockmass between source and receiver, our current approach is to simplify the situation somewhat: Picked arrivals are used as data, including points within a short window surrounding the pick, and long enough to contain the direct arrival pulse. Only those reflections which overlap the direct arrivals in time are modelled, and their modelled velocities are allowed to vary slightly to accommodate changes in velocity in the ground, as well as changes in elevation of the coal seams. Only coal seams between or immediately above or below the source and receiver are considered. This is because a raytracing algorithm is being used, and the number of possible reflected rays increases exponentially with the number of interfaces. The source is assumed, at this stage, to be a Brune pulse with only amplitude and pulse-width as parameters (no directional dependence).

In spite of these simplifications, the method is successful in providing consistent moment tensor fits to the waveform data in the vicinity of the picked $\mathrm{P}$ and $\mathrm{S}$ arrivals. The biggest problem currently remaining is that the error surfaces have multiple minima, and so, unless good starting parameters are chosen, the inversion algorithm frequently gets stuck in a local minimum. Since our microseismic surveys often record many thousands of events per day, it would be highly desirable to overcome this problem so that the procedure can be made fully automatic.

\section{CONCLUSIONS}

This paper has demonstrated that the shape of measured seismic waveforms in a coal environment is strongly affected by three factors: the presence of reflected and converted modes from coal seams; strong S-wave splitting; and nearfield effects. Attempts to invert seismic data for moment tensor parameters fail when these effects are not taken into account, producing inconsistent results which are unable to fit the data. Even thin coal seams, of half a metre to a metre thick, can have a drastic effect on waveform shapes for lowangle rays. This phenomenon explains the often-observed lack of strong SV arrivals in the microseismic data. Near-field effects, which are seldom taken into account, have been shown to have a significant effect on displacement waveforms, as well as potentially providing independent information on the shape of the source pulse.

\section{ACKNOWLEDGMENTS}

This work was jointly funded by ACARP and CSIRO. Thanks to Peter Hatherly and Xun Luo for discussions on all the seismic issues, to Winton Gale for discussions on the relationship between seismic stress drop and rock strength and to Art McGarr for suggesting that near-field effects may be present.

\section{REFERENCES}

Aki, K. and Richards, P.G. (1980) Quantitative Seismology: Theory and Methods: W.H. Freeman and Company, San Francisco.

Hatherly, P., Luo, X., McKavanagh, B., and Dixon, R. (1997) Seismic monitoring of ground caving processes associated with longwall mining of coal. In 4th International symposium on rockbursts and seismicity in mines. Krakow, Poland, pp. 121-124.

McGarr, A. (1992a) An impulsive component in the seismic moment tensor of a mining-induced tremor. Geophysical Research Letters. 19: 1579-1582.

McGarr, A. (1992b) Moment tensors of ten Witwatersrand mine tremors. PAGEOPH. 139: 781-800. 